\title{
Zur Struktur von Eskern in Schleswig-Holstein, unter besonderer Berücksichtigung des „Esker-Kames-Systems Forst Steinburg" in morphologischer Hochlage
}

\author{
Alf Grube
}

Abstract:

Different esker forms in Schleswig-Holstein were investigated as part of the geotop register of Schleswig-Holstein: purely glaciofluvial eskers, eskers with a core of till or glaciolacustrine sediments, beaded eskers ("Perlenschnur-esker"), till-covered eskers, cross-cutting eskers, crooked- and separated-parallel eskers as well as eskers with and without accompanying erosional channels. Eskers are associated with and/or genetically linked to drumlins or drumlinoid forms, adjoining till diapirs, ice marginal structures, kames or dead ice forms. An "esker-kames system" that is superimposed on an ice-marginal moraine that attains local heights of $+85 \mathrm{~m}$ a.s.l. is described in detail. The structure is morphologically recognizable as gravelly sand ridge situated on an elevated sandy-gravelly area. The structure can be subdivided into three parts: (A) a western area (esker) clearly subdivided by parallel single structures, (B) a central, high gravelly sand ridge (subglacial esker or crevasse fill) as well as (C) hilltop-shaped areas at the southeast end of the structure (kames). The esker structure (A) shows a central, large gravelly sand ridge with a width of $70 \mathrm{~m}$, a height of c. 8-10 m and a length of c. $220 \mathrm{~m}$ (covered with till) and two parallel smaller esker ridges which run to the south of the larger one. The genesis of this esker-kames structure on top of an ice-marginal moraine is discussed.

[On the structure of eskers in Schleswig-Holstein with special emphasis on the "esker-kames-system Forst Steinburg" in morphological top position]

Kurzfassung:

Die unterschiedlichen Esker-Formen Schleswig-Holsteins wurden für das Geotop-Kataster des Landes mittels Literatur- und Feldarbeiten neu bearbeitet: Esker i.e.S., Aufpressungs-Esker mit Kern aus Geschiebemergel oder Beckensedimenten, Perlenschnur-Esker, Till-bedeckte Esker, sich kreuzend überlagernde Esker, verwachsen- und getrennt-parallele Esker-Rücken sowie Esker mit und ohne begleitende Rinnenbildungen. Esker sind häufig räumlich vergesellschaftet bzw. genetisch verflochten mit Drumlins oder drumlinoiden Formen, angrenzenden Aufpressungsstrukturen aus Till, Eisrandlagen, Kames-Bildungen oder Toteis-Bereichen. Im Detail wird ein „Esker-Kames-System“ dargestellt, welches dem Scheitelbereich einer großen Eisrandlage mit örtlich +85 m NHN Höhe aufgesetzt ist. Die morphologisch als Kiessand-Rücken erkennbare Gesamtstruktur sattelt einem aufgewölbten, sandig-kiesigen Bereich auf. Die Struktur kann in drei Teile untergliedert werden: (A) einen flacheren, deutlich durch parallele Einzelstrukturen gegliederten westlichen Bereich (Esker), (B) einen zentralen, hohen Kiessand-Rücken (Esker oder Spaltenfüllung) sowie (C) kuppenförmige Bereiche am Südostende der Struktur (Kames). Die Esker-Struktur (A) zeigt neben einem zentralen, großen Kiessand-Rücken mit 70 m Breite, einer Höhe von ca. 8-10 m und einer Länge von ca. $220 \mathrm{~m}$ (mit Till bedeckter-Esker) zwei parallele kleinere Esker-Rücken, die südlich des großen verlaufen. Die Genese der Esker-KamesStruktur in Randlagen-Top-Position wird diskutiert.

Keywords: $\quad$ Esker, Os, Kames, Crevasse Filling, Weichselian

Address of author: Dr. A. Grube, Geologischer Dienst, Landesamt für Landwirtschaft, Umwelt und Ländliche Räume des Landes Schleswig-Holstein, Abt. Geologie und Boden, Hamburger Chaussee 25, 24220 Flintbek. E-Mail: alf.grube@llur.landsh.de

\section{Einleitung}

Schleswig-Holstein besitzt aufgrund seiner Entstehungsgeschichte eine beeindruckend vielfältige Geologie und Geomorphologie (GRUBE 2008). Viele Landschaftsformen sind noch nicht genauer wissenschaftlich untersucht. Die unterschiedlichen vorhandenen Esker-Formen SchleswigHolsteins wurden für das Geotop-Kataster des Landes neu überarbeitet. Eine besondere Struktur der Inlandvereisung (Weichsel-Kaltzeit) im Forst Steinburg (Landkreise Stormarn und Herzogtum Lauenburg) wurde in diesem Rahmen geologisch näher untersucht.

Im Folgenden wird der Begriff Esker verwendet, da er definiert und international gebräuchlich ist. Der Begriff Os ist dagegen undeutlicher, im Skandinavischen wird der Begriff Rullsten-Os verwendet, während der Begriff Os weiter gefasst ist und z.B. Festgesteins-Strukturen beinhaltet. Im Deutschen ist der Begriff Wallberg speziell in der älteren wissenschaftlichen sowie populärwissenschaftlichen Literatur verbreitet, auch dieser wird nicht einheitlich verwendet.

Kames sind eine für Niedertaulandschaften typische glazigen gebildete geomorphologische Form. Der Begriff Kames stammt aus dem Schottischen (kaim = Rücken mit steilen Flanken) und wurde von JAMIESON (1874) eingeführt. Kames entstehen während der Abtauphase des Gletschers (vgl. Brodzikowski \& VAN LooN 1991). Kames-Kuppen wurden aus Sedimentfüllungen in Becken, Wannen usw. unter, im oder auf dem Eis gebildet, die nach Abschmelzen durch Reliefumkehr ihre rundlich-gewölbte Form erhalten. Häufig dominieren dabei feinkörnige Schmelzwasserbildungen (Feinsande), die durch langsam fließende Wässer über längere Zeiträume in Hohlformen ablagert werden. 


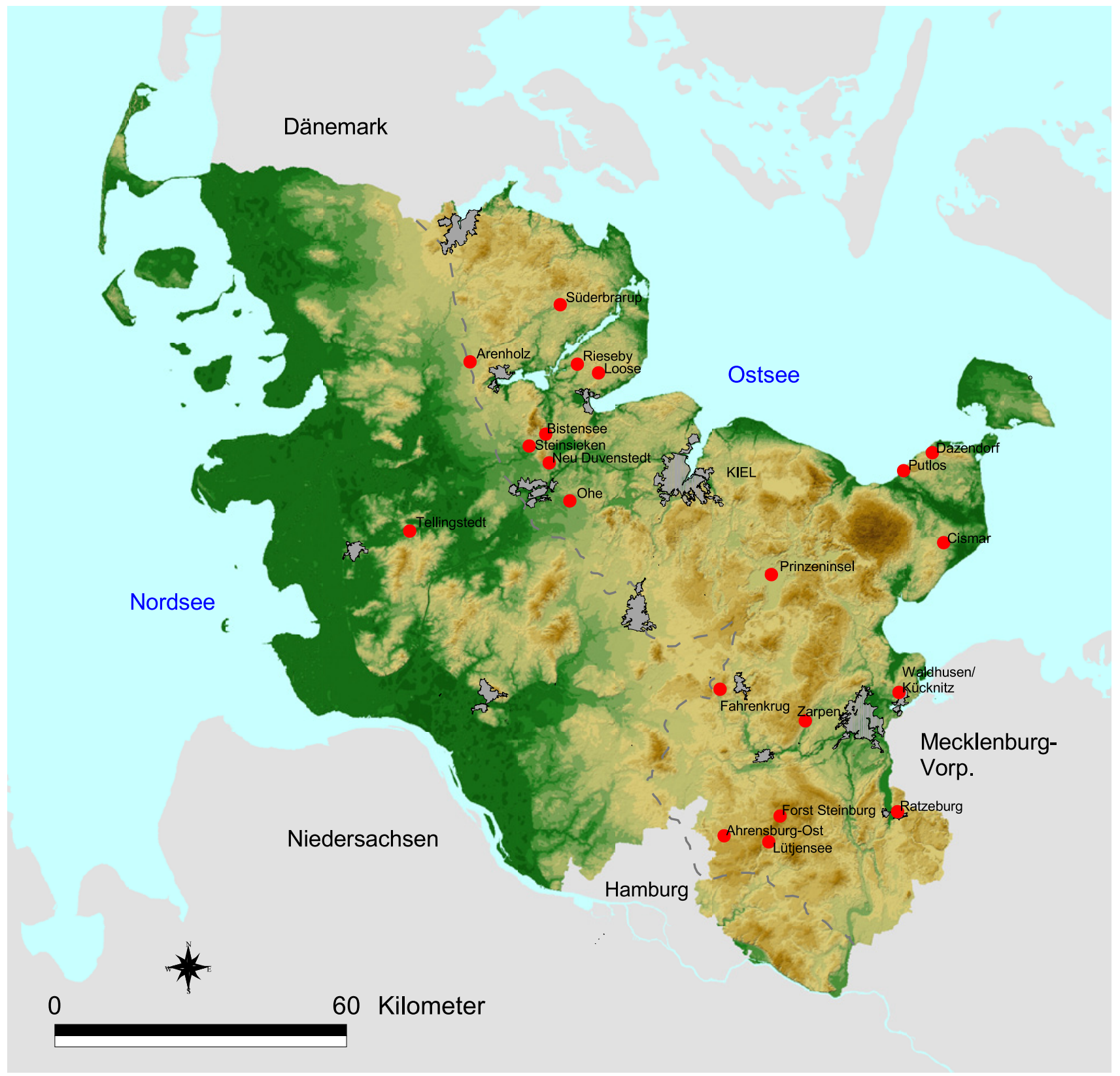

Abb. 1: Lageplan der im Text erwähnten Esker-Strukturen (Grundlage: Landesvermessungsamt SH). Gestrichelt: Maximale Ausdehnung der WeichselVereisung.

Fig. 1: Plan with esker structures mentioned in the text (basis: Landesvermessungsamt SH). Dashed line: maximum of Weichselian glaciation.

\section{Zur Bildung von Eskern in Schleswig-Holstein}

Esker sind langgestreckte, oft gewundene, wallartige Geländerücken. Sie werden aus Sanden und Kiesen mit komplexer Internstruktur aufgebaut, lokal finden sich Till-Einschaltungen oder Beckenablagerungen. Aufpressungs-Esker können einen Kern aus Till besitzen, lokal können Tone in die Bildung einbezogen sein, wie z.B. Holsteinzeitliche Tone (vgl. Schulz 1970). Die internen Verstellungen gehen auf glazi-tektonische Stauchungen, Setzungen aufgrund des Niedertauens des umgebenden, stabilisierenden Eises, Austauen von Toteis usw. zurück. Bei Aufpressungs-Eskern ist durch die vertikal von unten gerichtete Druckfront mit entsprechenden Störungen zu rechnen. Esker entstehen in sub-, in- oder supraglaziär angelegten Schmelzwassertunneln bzw. -spalten, welche häufig die Fortsetzung von
Spalten des supra- oder inglaziären Gletscherbereiches sind (z.B. Arenholzer Esker (Abb. 1); WüNNEMANN 1990). Die heute noch erhaltenen Esker entstanden in den Spätoder Abschmelzphasen der Vereisungen. Sie finden sich ganz bevorzugt in Tal-Bereichen, im ehemaligen Randbereich von Gletschern. Sie blieben nur unter besonderen Bedingungen erhalten und sind ganz überwiegend weichselkaltzeitlichen Alters. Selten besitzen Esker oder Esker-ähnliche Strukturen ein Saale-kaltzeitliches Alter wie bei einer fraglichen Esker-Struktur bei Tellingstedt (GRUBE 2011). Örtlich finden sich Unterbrechungen, die aufgrund ihrer meist rundlichen Form als Os-Augen bezeichnet werden. Sie gehen auf in den Spalten bzw. Tunneln liegen gebliebene Eisblöcke zurück. Häufig treten parallel zu den Eskern verlaufende Rinnen auf, die durch Toteis-Bildungen oder durch Strömungswalzen (vgl. ARIo 1977) entstanden sind. 
Rechtwinklig abzweigend können Ablagerungen in ehemaligen Querspalten abgelagert worden sein, die aus Kiessanden oder Till bestehen können.

Insgesamt ist das Spektrum der auftretenden Esker-Formen offenbar kleiner als in Skandinavien (vgl. LuNDQVIST 1979). Esker i.e.S. sind verhältnismäßig selten. Sie mögen früher häufiger vertreten gewesen sein, bekanntermaßen sind leider viele dieser Kies-Sand-Strukturen durch Sandentnahmen zu Bauzwecken zerstört worden. $\mathrm{Zu}$ den Eskern i.e.S. gehören z.B. die Strukturen bei Arenholz und Süderbrarup (WüNNEMANN 1990), Neu Duvenstedt sowie Putlos (Grube 2011). Daneben finden sich komplexe EskerFormen, bei denen sich Esker kreuzend überlagern, bedingt durch entsprechende Spaltenbildungen in verschiedenen, übereinander liegenden Gletscher-Niveaus. Hierzu gehört das Esker-System in Ahrensburg-Ost / Stellmoorer Tunneltal (KöLling \& SchlüTER 1988). Auch mehrere Strukturen im Bereich der Eiszerfallslandschaft am Lütjensee gehört in diese Gruppe (Grube 2011). Treten Parallel-Strukturen auf, so können „verwachsene“ und „getrennt-parallele“ Esker unterschieden werden. Am Esker-System Waldhusen-Kücknitz sind Rücken des Eskers miteinander „verwachsen“. Getrennt-parallele Strukturen sind z.B. am Esker-KamesSystem Forst Steinburg (dieser Aufsatz) vorhanden. Seltener besitzen Esker in Schleswig-Holstein einen Mantel aus Till, der unmittelbar ihre sub- oder inglaziäre Entstehung belegt. Eine relativ dünne Till-Decke ist am Esker-KamesSystem Forst Steinburg zu finden (vorliegender Aufsatz), im Bereich Loose ist auf einer Struktur eine mehrere $\mathrm{m}$ mächtige Abdeckung vorhanden (GRUBE 2011). Ein- oder beidseitig parallel verlaufende, heute meist vermoorte und morphologisch nicht oder kaum zu erkennende, rinnenförmige schmale Randsenken sind bei den Eskern in Ohe (EgGers 1934, Strehl 1993) und Ahrensburg-Ost (Kölling \& SCHLÜTER 1988) kartiert worden. Örtlich können diese im Liegenden der üblicherweise abdeckenden limnischtelmatischen Ablagerungen mit Beckenablagerungen gefüllt sein, wie bei Fahrenkrug (GRUBE 2011). Der vermutete Esker bei Zarpen ist eine sehr große und heterogen zusammengesetzte Struktur, die parallel zur Umrandung des Lübecker Beckens verläuft und somit eine Spaltenfüllung darstellen könnte.

Bei Aufpressungs-Eskern ist vorwiegend bindiges Material von unten in einen subglaziären Tunnel aufgepresst worden. Ein Kern aus Till ist bei den Strukturen Dazendorf (vgl. SeIfert 1953), Cismar (Grube 2011) und im nördlichen Teil des Eskers von Waldhusen/Kücknitz (STRUCK 1918; vgl. Gagel 1923, Ohnesorge 1928) vorhanden. Auch die Prinzeninsel im Plöner See (Grube 2011) könnte entfernt zu dieser Gruppe gehören. Der Esker von Waldhusen/Kücknitz (GRUBE 2011) zeigt im südlichen Abschnitt als Besonderheit einen Kern aus Beckenschluffen. Eine seltene Form der Esker in Schleswig-Holstein sind PerlenschnurEsker. Derartige Strukturen treten bei Bistensee (STREHL 2006), und bei Rieseby auf, hier allerdings morphologisch nicht sehr prägnant (GRUBE 2011).

Esker sind häufig mit Drumlins, drumlinoiden Formen, angrenzenden Aufpressungsstrukturen aus Till, Kames-

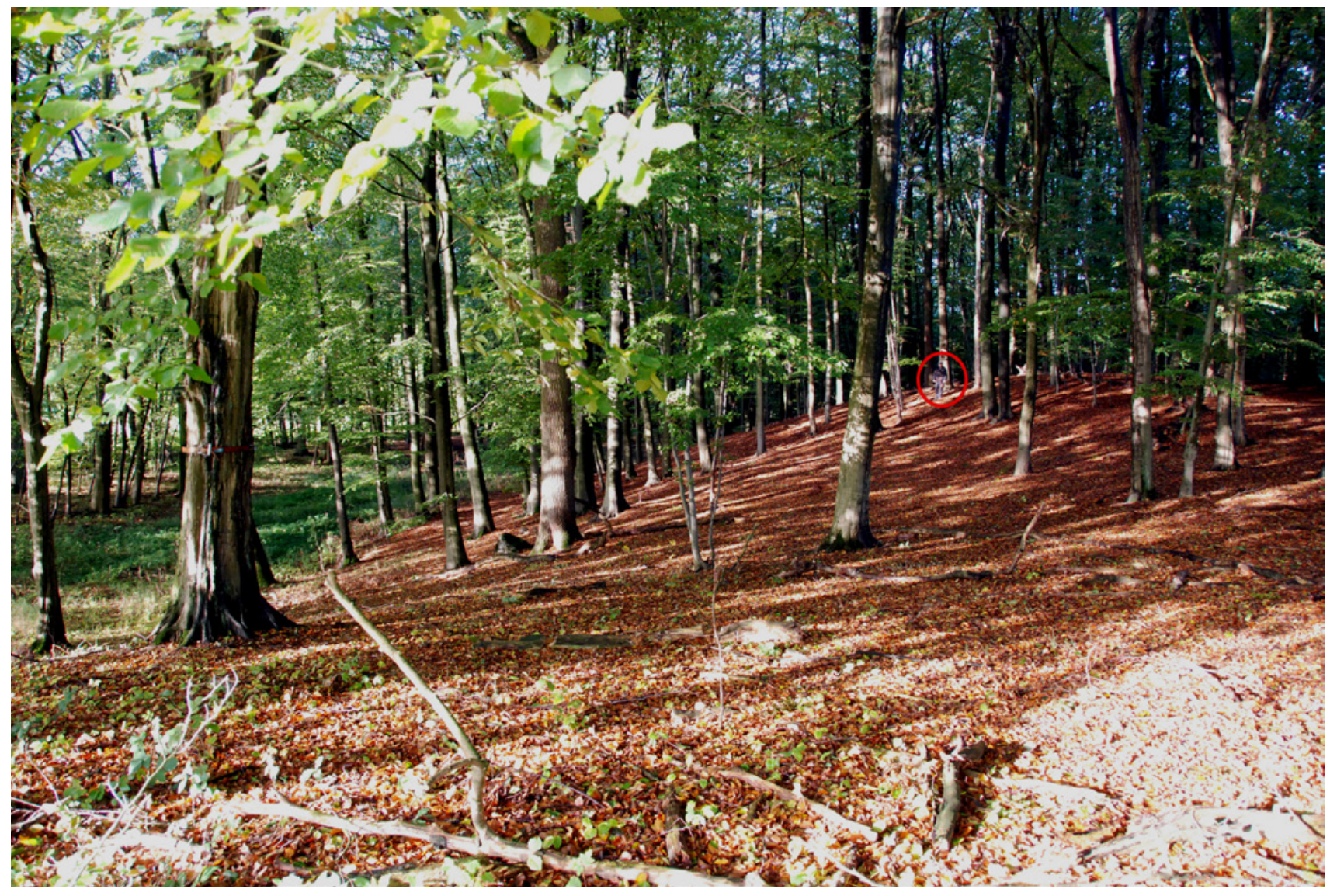

Abb. 2: Esker-Bereich (Teil „A“; Person im Kreis als Maßstab). Standpunkt R 3593320, H 5953050, Blickrichtung nach Westen.

Fig. 2: Esker (part „A“; person for scale). Position: $R$ 3593320, H 5953050, view towards west. 
Bildungen und Toteisbereichen räumlich vergesellschaftet bzw. genetisch verflochten. Sie zeigen teilweise eine Parallelität mehrerer Esker-Rücken, zumindest in Teilbereichen. In Verbindung zu einer Esker-Struktur auftretende KamesBildungen, die sich z.B. durch eine breitere Form sowie eine feinsandige Zusammensetzung auszeichnen, sind $\mathrm{u}$. a. bei den Eskern in Rieseby und Forst Steinburg vorhanden.

\section{Das „Esker-Kames-System Forst Steinburg“}

\subsection{Umgebung der Struktur}

Die Struktur liegt in der südlich der Umrandung des Lübecker Beckens. Nach der alten geologischen Karte (Blatt 2028 Eichede, Bearbeiter: P. Range, 1927-1928 und Blatt 2328 Trittau; Bearbeiter: P. Range, 1925-1926) kann der gesamte Bereich der Eisrandlage als glazitektonisch überprägt angesehen werden. Die Verstellungen sind teilweise massiv, u.a. sind Schuppen aus miozänen Ablagerungen im dem Höhenrücken bei Eichede bekannt geworden (vgl. HALsKe 1924).

In der o. g. preußischen geologischen Karte ist das hier dargestellte Gebiet als 3,0 km lange und bis zu $300 \mathrm{~m}$ breite Zone mit kiesig-sandigen Ablagerungen eingetragen. Westlich schließen sich nach den geologischen Übersichtskarten in der Hohlform zwischen Todendorf und Oetjendorf glazifluviatile Ablagerungen an, die als kleine Binnensanderbereiche zu interpretieren sind. Westlich und nördlich dieser rinnenartigen Schmelzwassersandbildungen finden sich große glazi-limnische Becken, die beim Niedertauen des Gletschers entstanden sind, ggf. zeitgleich mit der Bildung des Esker-Kames-Systems. Am südlichen Ortrand von Mollhagen befindet sich ein kleinerer, morphologisch deutlich im Gelände erkennbarer Kiessand-Rücken, der als Esker zu deuten ist. Er ist weitgehend bebaut.

\subsection{Esker-Kames-Struktur im Forst Steinburg}

Die Entdeckung und Anregung zur Bearbeitung des vermuteten Esker-Bereiches geht auf Herrn Peter Jacobi (Delingsdorf) zurück, dem für die Mitteilung herzlich gedankt sei. Leider erfolgte, trotz entsprechender früher Bemühungen u. a. von Carl Duve (Schreiben an den Kreis Stormarn von 1961), eine intensive Überprägung des Bereiches durch Sand- und Kiesabbau.

Im Folgenden wird von einem „Esker-Kames-System“ gesprochen, da der Bereich durch Teilgebiete verschiedener Genese zusammengesetzt ist. Die Struktur befindet sich auf dem Scheitelbereich einer großen Eisrandlage (Abb. 3), die örtlich $+85 \mathrm{~m} \mathrm{NHN}$ erreicht. Die Struktur hat eine Länge von ca. $3 \mathrm{~km}$ und eine Breite von bis zu ca. $1 \mathrm{~km}$. Größere Teile der Gesamtstruktur sind leider dem Kiesabbau zu Opfer gefallen, so dass nur ein Stück seine ursprüngliche Oberflächenform aufweist. Für die abgebauten Flächen sind der Aufbau und die Entstehungsgeschichte leider kaum noch zu belegen. Nach den alten preußischen topographischen Karten von 1790 sind in den inzwischen abgebauten Bereichen einzelne, größere Höhenrücken zu erkennen. Die alten Karten geben die Feinmorphologie leider kaum wieder, so dass Unsicherheiten bezüglich der ursprünglichen Morphologie des Gesamtgebietes bestehen.

Die morphologisch als Kiessand-Rücken erkennbare Ge- samtstruktur sattelt einem aufgewölbten, sandig-kiesigen Bereich auf, der morphologisch durch ca. 0,5 km weite „Vorsprünge" westlich und östlich der Randlage in Erscheinung tritt. Der Kiessand-Rücken kann dabei grob in drei Teile untergliedert werden: (A) einen flacheren, deutlich durch parallele Einzelstrukturen gegliederten westlichen Bereich, (B) einen zentralen, hohen Rücken sowie (C) in kuppenförmige Bereiche am Südostende der Struktur (Abb. 4).

\section{(A) Westlicher, differenzierter Bereich mit mehreren par- allelen Kiessand-Rücken}

Dieser Bereich zeigt neben einem zentralen, großen Kiessand-Rücken zwei weitere kleinere, die südlich des großen verlaufen (Abb. 3).

Der zentrale, große Kiessand-Rücken ist durch den Kiesabbau an seiner Nordflanke beeinträchtigt. Die heutige Steilkante ist durch einen Teilabtrag des Rückens bedingt. Er besitzt eine Breite von ca. $70 \mathrm{~m}$ und eine Höhe von ca. 8-10 m. Er ist in der heutigen Form noch auf einer Länge von ca. $220 \mathrm{~m}$ erhalten. Auf dem östlichen Ende läuft er aus und endet kurz vor einer Auffüllung. An seinem westlichen Ende ist er durch den Kiesabbau bis direkt an die Kreisgrenze abgetragen worden. Durch eine kleine, ca. $20 \mathrm{~m}$ breite Niederung getrennt, verläuft südlich der großen Struktur ein kleinerer Rücken, der auf einer Länge von ca. 200 m, eine Breite von 15-20 m und eine Höhe von ca. $1 \mathrm{~m}$ zeigt. Diese flache „mittlere“ Struktur setzt sich auf stormarner Gebiet weiter fort. Im Bereich des Hauptrückens wurden in zwei Bohrungen unter einem Geschiebedecksand (westlicher Teil) bzw. einem ca. 1,5 m mächtigen Geschiebelehm ca. 5,0 m bzw. 5,5 m mächtige Kiessande erbohrt (Abb. 6). Diese Sande und Kiese sind nur oberflächennah entkalkt (ca. 2,6 m u. GOK). Das Liegende der Kiessande in beiden Bohrungen wird durch Geschiebelehm und -mergel gebildet.

Die ursprüngliche Fortsetzung des großen Kiesrückens von der lauenburgischen auf die westlich gelegene stormarner Seite ist unklar (ursprüngliche Länge bis zu ca. 700 m), da die Struktur hier vollständig durch den Kiesabbau abgetragen worden ist. Nach der preußischen topographischen Karte dürfte er etwas flacher als seine inzwischen abgetragene, östliche Fortsetzung gewesen sein. Ggf. könnten hier genauere geologische und geophysikalische Untersuchungen der verbleibenden Sedimentschichten an der Abbausohle zu einer Klärung führen, die jedoch späteren Untersuchungen vorbehalten bleiben müssen. Vermutlich waren Teile des westlichen Esker-Teiles ebenfalls mit Till bedeckt.

Auf Stormarner Seite sind insgesamt drei parallele Rückenstrukturen erkennbar. Dabei ist eine Abnahme der Größe von Nord nach Süd feststellbar. Die breite Hauptstruktur, die auf Lauenburger Seite stehen geblieben ist, ist hier vollständig abgetragen, zumindest die übertägigen Teile. Die Fortsetzung der südlich gelegenen, „mittleren" Struktur hat eine Länge von ca. $300 \mathrm{~m}$, eine Höhe von 3 bis $4 \mathrm{~m}$ und eine Breite von ca. 25-30 m. Hierbei sind die nördlichen Teile und auch die Flankenbereiche durch Sandgewinnung gestört. Westlich dürfte sich dieser Rücken noch weiter nach Westen fortgesetzt haben, der ungefähre Verlauf ist auch nach dem Kiesabbau noch zu erkennen. Wiederum durch eine kleine Niederung getrennt verläuft südlich ein weiterer Rücken. Dieser setzt ungefähr an der 


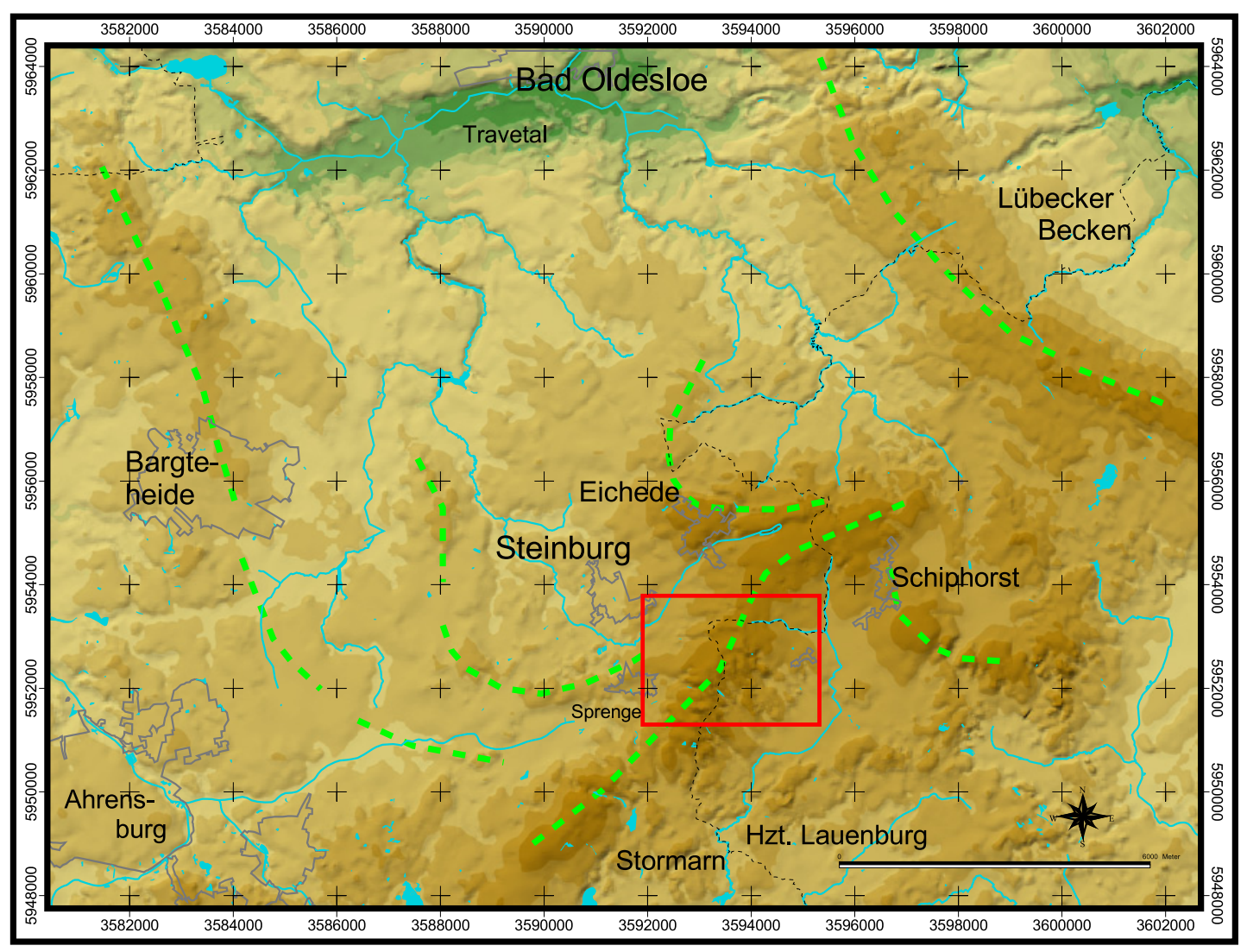

Abb. 3: Haupteisrandlagen vor dem Hintergrund des Digitalen Geländemodells (Landesvermessungsamt SH); mit Umrandung des engeren Untersuchungsgebietes.

Fig. 3: Main ice marginal position on digital terrain model (Landesvermessungsamt SH) with contour of closer investigation area.

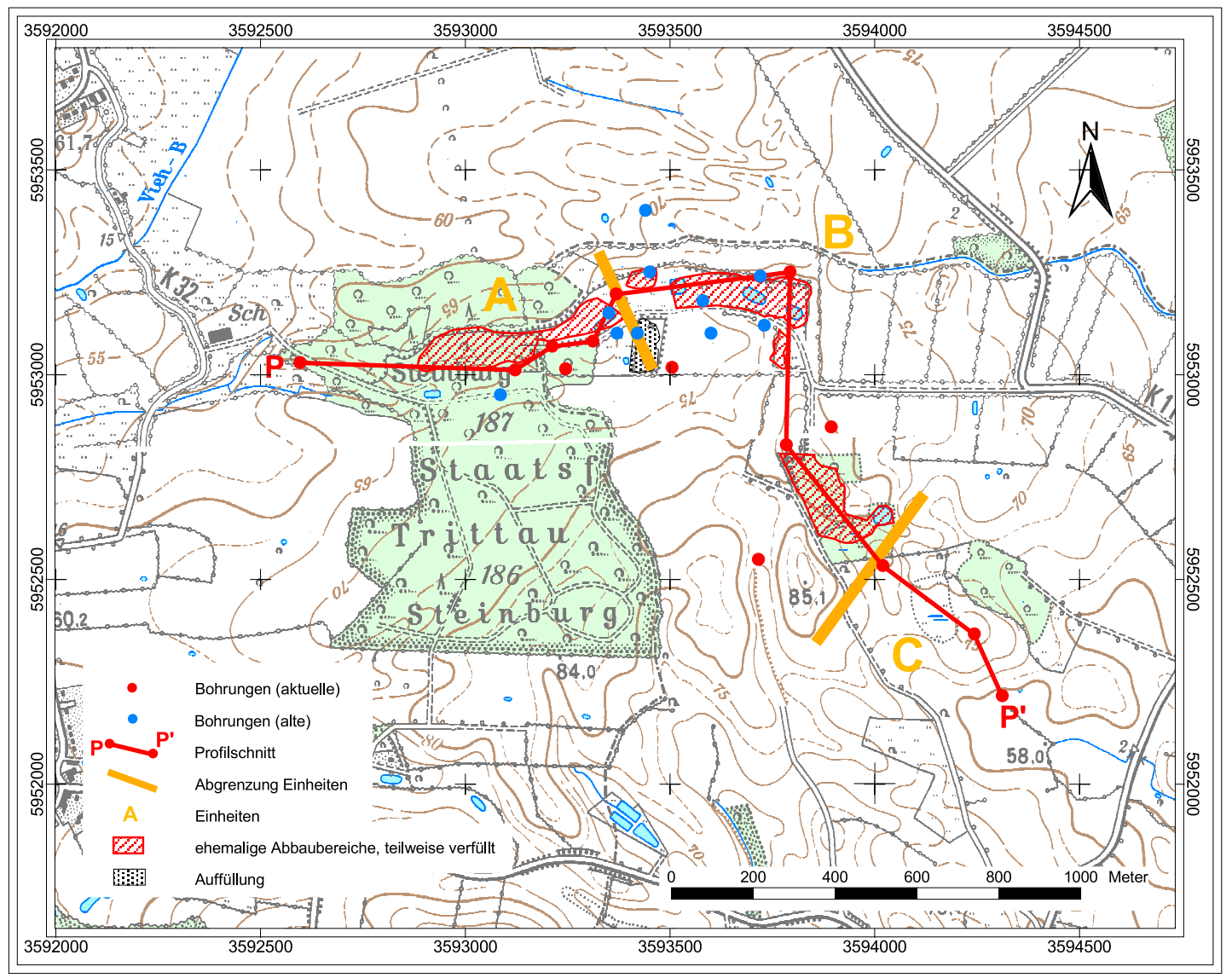

Abb. 4: Esker-Kames-System mit Teilbereichen A, B und C; Bohrungen, Lage des Profilschnittes, Abbauflächen und Auffüllung (TK25).

Fig. 4: Esker-Kames-structure with parts A, B and C; position of drillings, cross section, excavation sites and dump (TK25). 
Kreisgrenze an, läuft ebenfalls in West-Ost-Richtung und ist ca. $150 \mathrm{~m}$ lang, ca. 1-2 $\mathrm{m}$ hoch und ca. 25-30 m breit. Dieser verhältnismäßig flache Rücken hat eine Länge von ca. $70 \mathrm{~m}$ und nicht die typische Wallform eines Eskers.

Am Südrand der Struktur „A“, ansetzend am östlichen Ende der gut erhaltenen Eskerstruktur und fortlaufend an der abgebauten Struktur, findet sich eine parallele Rinnenbildung, die heute an der Erdoberfläche nicht mehr zu erkennen ist (Fastebene), da sie mit Torfen verfüllt ist. Die Mudden werden mehr als 1,5 m mächtig, im Liegenden folgt ein ca. $1 \mathrm{~m}$ mächtiger Beckenschluff.

\section{(B) Zentrale lang gestreckte Vollform}

Hierbei handelt es sich um einen breiten Kiesrücken, der überlappend mit dem unter „A“ beschriebenen großen Rücken, aber ca. $100 \mathrm{~m}$ nach Norden versetzt beginnt, dann ca. $400 \mathrm{~m}$ in West-Ost-Richtung verläuft, um dann rechtwinklig nach Süden abzuknicken. Nach weiteren ca. $400 \mathrm{mn}$ biegt er nach Südsüdost ab und endet hier nach ca. $400 \mathrm{~m}$ (Gesamtlänge ca. 1,2 km).

Während der erste Teil der zentralen Vollform rechtwinklig zu der unterlagernden Eisrandlage orientiert ist, ist der letzte Teil parallel zu dieser bzw. zu einzelnen parallelen Höhenrücken auf diesem angelegt (Abb. 4). Die zentrale Vollform ist weitgehend abgebaut worden. Stehen geblieben ist ein ca. $300 \mathrm{~m}$ langes Teilstück. Nach der alten preußischen topographischen Karte von 1790 hatte der singuläre Höhenrücken eine Höhe von bis zu mehr als $10 \mathrm{~m}$. Er ist bis zu $150 \mathrm{~m}$ breit, zeigt im mittleren Teil eine Art Einschnürung, in diesem Bereich ist der Rücken nur ca.
$80 \mathrm{~m}$ breit. Nach dem rechtwinkligen Abknicken nach Süden wird die Struktur unregelmäßiger, erreicht lokal eine Breite von mehr als $200 \mathrm{~m}$. Hier wird mit mehr als $+88 \mathrm{~m}$ NHN auch seine größte Höhe erreicht.

Die ehemaligen Kiesgewinnungsbereiche, die Sohle des Abbaus, stimmt in etwa mit der Basisfläche der Struktur auf der Eisrandlage überein (Abbautiefe häufig 5-8 m), zeigen einen außergewöhnlich hohen Anteil an Blöcken und Findlingen. Selbst unter der Annahme, dass nach dem Kriege technisch bedingt größere Blöcke und Findlinge nicht gut verarbeitet werden konnten (z. B. durch Verwendung im Gartenbaubereich oder Zerkleinern durch Brecher), so ist doch von einem sehr hohen Anteil der Grobfraktion (Kies bis Findlinge) auszugehen.

Auf eine parallel zum ehemaligen Höhenrücken verlaufende Rinnenbildung wurde bereits oben eingegangen (vgl. Abschnitt „A“).

Am südlichen Ende des Strukturabschnittes „B“ setzen beiderseits des zentralen Kiesrückens zwei bis zu mehr als $5 \mathrm{~m}$ tief eingeschnittene Abflussrinnen ein, durch die radialstrahlige Entwässerung der Schmelzwässer erfolgte (Abb. 7). Die tiefste Rinne verläuft in Nord-Süd-Richtung, eine weitere deutliche Rinne in NNW-SSE-Richtung. Nach Südosten und Osten sind drei weitere kleinere Abschlussbahnen vorhanden. Diese setzen sich im Abschnitt „C“ weiter fort (siehe dort).

(C) Kuppenförmige Bereiche am Südost-Ende der Struktur Die Folge schließt dann mit einer großen, lang gestreckten Kuppenlandschaften ab, die fast vollständig aus Fein-

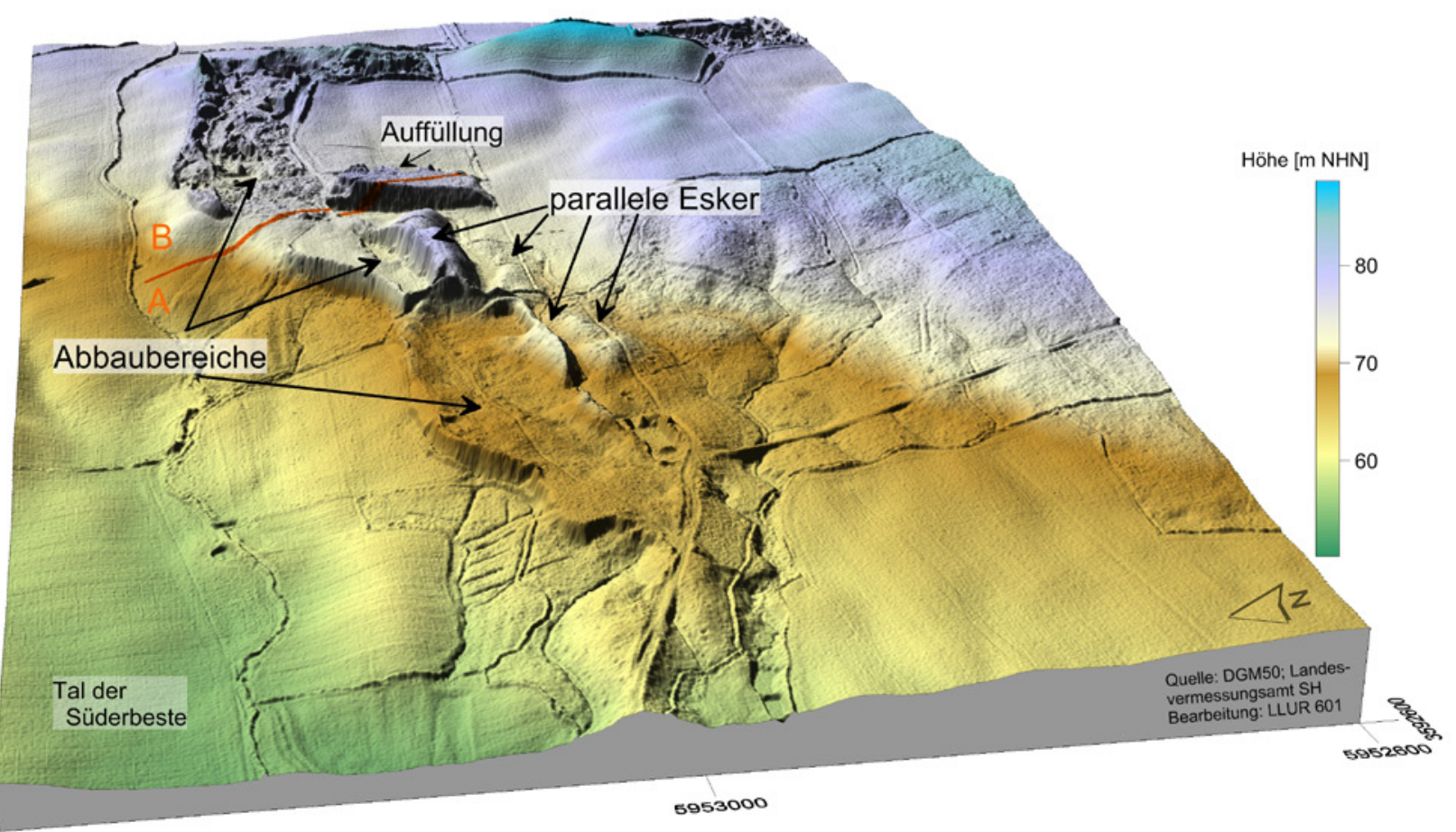

5953400

Abb. 5: Digitales Geländemodell eines Teils der Struktur (Teile A und B).

Fig. 5: Digital terrain model of part of the structure (parts $A$ and B). 
sand besteht (Abb. 6). Dieser knapp 500 m lange Strukturabschnitt ist durch den Kiesabbau nicht beeinträchtigt, vermutlich wegen der geringen Kiesanteile. Der Feinsand erreicht eine Mächtigkeit von fast $10 \mathrm{~m}$, einschließlich einer geringmächtigen Geschiebemergeleinschaltung, die als Fließ-Till gedeutet werden kann. Im Liegenden der Feinsande sind mit verhältnismäßig direktem Übergang Geschiebelehme und -mergel vorhanden. Diese lang gestreckte Kuppe, die den morphologischen Abschluss der Eisrandlage zur östlich benachbarten Niederung der Schönau bildet, verläuft etwa in gleicher Orientierung wie der unter „B“ beschriebe Strukturteil, knickt jedoch nach Osten ab.

Die unter „B“ beschriebenen Abflussrinnen (Abb. 7) setzen sich weiter nach Südosten fort. Sie verlaufen westlich bzw. östlich parallel zum Bereich „C“. Eine weitere Abflussbahn ist direkt westlich der Kames-Kuppe vorhanden. Die Abflussrinnen verästeln sich teilweise weiter und reichen bis in die benachbarte ausgedehnte Hohlform hinein, die mit mächtigeren Schmelzwassersanden flächenhaft aufgefüllt ist.

\section{Diskussion zur Entstehung der Struktur}

Die beschriebene Struktur ist aus verschiedenen Sedimentations- und Erosionsformen zusammengesetzt, die als Teile eines Entwässerungssystems einer Gletscherzunge entstanden. Trotz intensiven Kiesabbaus sind Teile der natürlichen Morphologie gut erhalten.

Bei den parallelen Kiessand-Rücken (Strukturteil „A“) handelt es sich um parallele Esker. Der große Rücken ist verhältnismäßig breit (Originalbreite vor Teilabbau ca. $80 \mathrm{~m}$ ). Er gehört damit zu den sehr großen Eskern Norddeutschlands, z.B. vergleichbar mit dem Esker von Waldhusen-Kücknitz. In Skandinavien sind bedeutend größere
Strukturen beschrieben worden. Die Till-Bedeckung des Kiessandrückens zeigt, dass dieser Teil der Struktur unter dem Eis gebildet worden sein muss. Weiterhin spricht hierfür auch die Bildung einer parallelen tieferen Rinne im Sinne AARIo's (1977) am Südrand dieses Strukturteils, die durch Strudelbildungen im Eistunnel zustande kommt (siehe Abschnitt 2). Speziell die parallelen flacheren Esker-Strukturen legen eine subglaziäre Entstehung nahe. LUNDQVIST (1979) beschreibt parallele Esker, die in Schweden verbreitet sind. Häufig sind hierbei drei parallele Esker-Strukturen (TANNER 1915, zitiert in LUNDQVIST 1979). Dieses könnte nach LuNDQvist mit dem Einfluss von Toteis zusammenhängen.

Bei der Zentralen Vollform (Strukturteil „B“) ist die Internstruktur aufgrund des erfolgten Abbaus nicht mehr sichtbar. Zeitzeugen oder eine Dokumentation der Abbaubereiche (Photos usw.) liegen nicht vor, zumal der Abbau bereits lange zurück liegt. Der flache Abbau spricht gegen das ehemalige Vorhandensein einer Till-Decke, da die Gewinnung von Sanden und Kiesen in diesem Fall nicht wirtschaftlich gewesen sein dürfte. Es könnte sich sowohl um eine Esker-, als auch um eine subaerische Spaltenfüllung handeln. Für eine Spaltenfüllung sprechen die gegenüber der westlich gelegenen Esker-Struktur deutlich höhere Aufragung, das Fehlen paralleler Voll- und Hohlformen (siehe oben) und die Häufung groben Materials. Der große Anteil an Blöcken könnte für eine Art Blockpackung sprechen, also eine Ansammlung von Geröll, die die Schmelzwässer in der Nähe des Gletschertores abgelagert haben. Für die subglaziäre Bildung als Esker spricht die Nachbarschaft zum westlich gelegenen Esker sowie die strukturparallele Rinnenbildung.

Bei der südlichen Vollform (Strukturteil „C“) handelt es sich um eine Kames-Bildung (Kames-Kuppen). Sie entstand

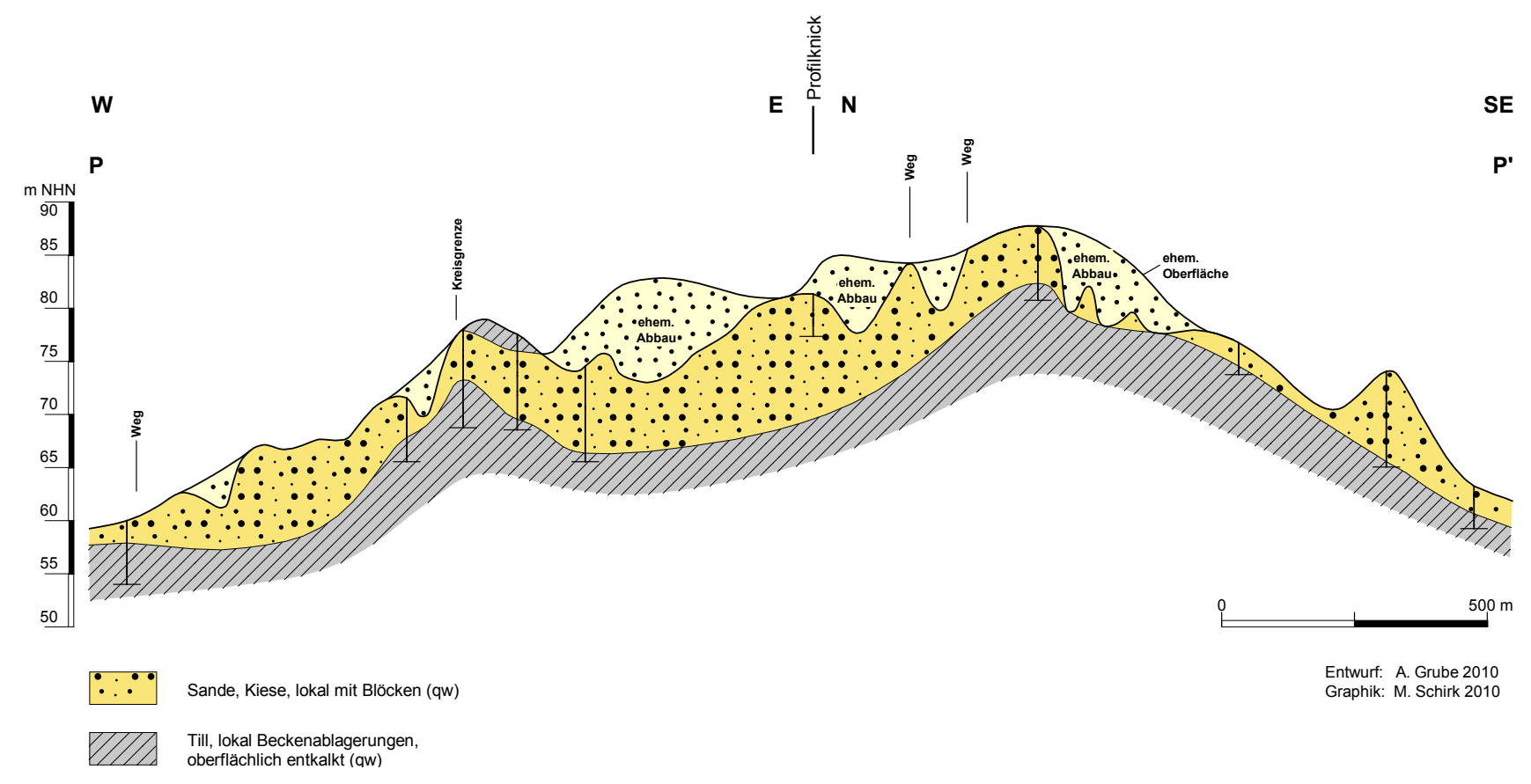

Abb. 6: Profilschnitt längs des Esker-Kames-System. Die ursprüngliche Morphologie wurde der preußischen topograph. Karte entnommen.

Fig. 6: Cross section along the esker-kames-structure. The original morphology was retained from the prussion topographical map. 


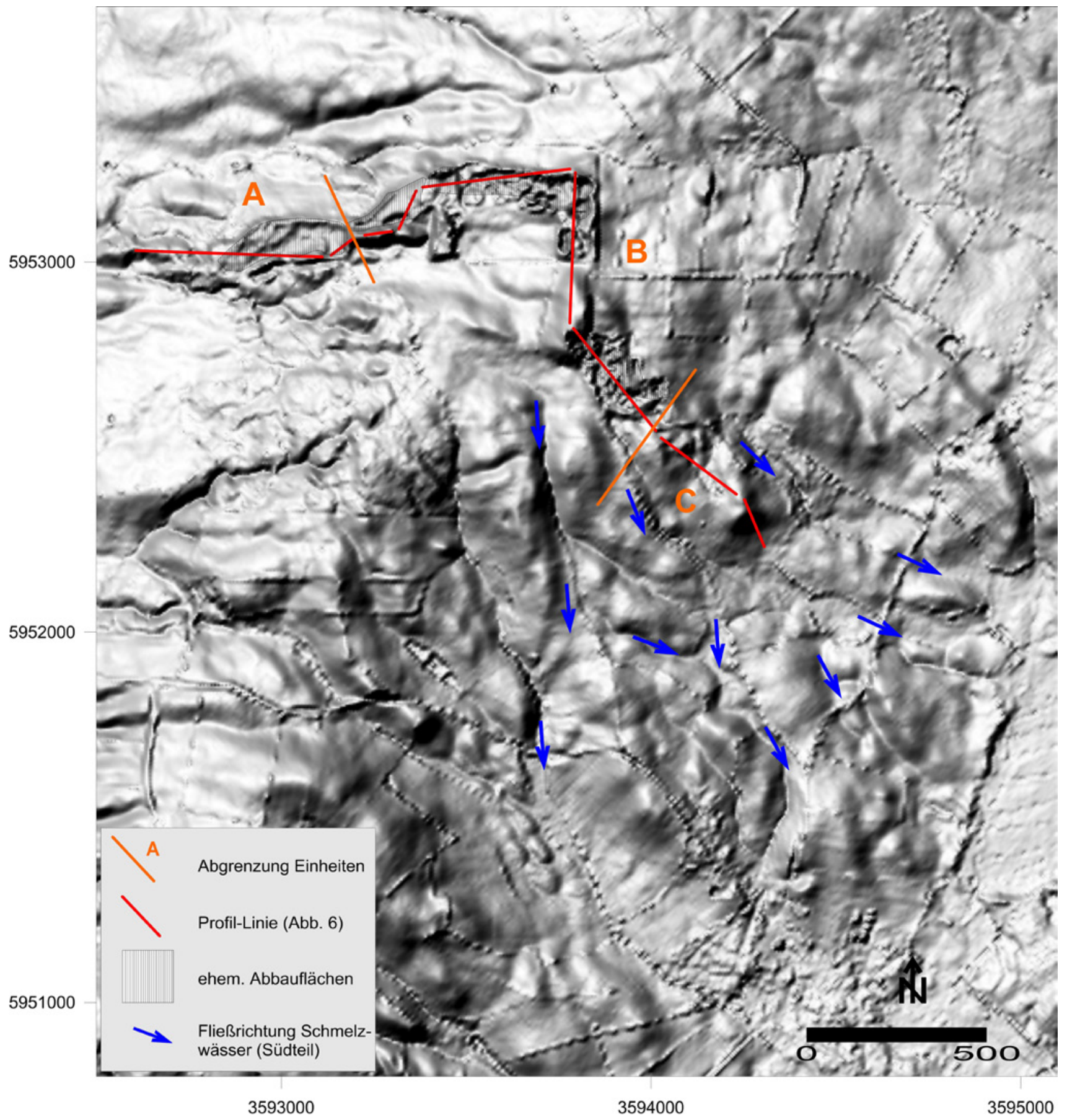

Abb. 7: Digitales Geländemodell des glaziären Abfluss-Systems (DGM5, Landesvermessungsamtes Schleswig-Holstein).

Fig. 7: Digital terrain model of the glacial drainage system (DGM5, Landesvermessungsamt Schleswig-Holstein).

am Ende der Struktur. Vorstellbar ist, dass eine große Eisspalte vor dem Gletscherrand bestand, bei der im zentralen Strukturbereich das gröbere Material geschüttet, und das feinere Material am äußeren Rand sedimentiert wurde. Auch eine subglaziäre Genese kann nicht ausgeschlossen werden. Der Übergang von Eskern und Spaltenfüllungen in Kames oder Sanderflächen ist eine nicht seltene Erscheinung in Norddeutschland.

Das „Esker-Kames-System“ liegt als besondere geologische Struktur auf einer großen Eisrandlage der WeichselKaltzeit. Dieser ca. $2 \mathrm{~km}$ breite Höhenrücken verläuft in NNE-SSW-Richtung etwa von Schiphorst nach Lütjensee.
Es befindet sich an einem Ansatzpunkt bzw. im Zwickel zu einer weiteren loben-förmigen Eisrandlage, die von Sprenge aus in westsüdwestlicher Richtung nach Todendorf verläuft, hier nach Nordnordwest umschwenkt und nach Hammoor verläuft. Weitere lobenförmige Eisrandlagen befinden sich ca. 1,5 km südlich sowie nördlich bei Eichede.

Das Esker-Spaltenfüllung-Kames-System ist im Randbereich eines Gletscherbereiches gebildet worden. Die Struktur läuft zentral über den Kamm der großen Eisrandlage. Dabei ist eine Verlaufsveränderung von einem Ost-WestStreichen im westlichen Teil zur einer Nordwest-SüdostErstreckung im östlichen Teil vorhanden. Die Anlage der 
Struktur hängt mit der Entwässerung der zugehörigen Gletscherzunge zusammen. Aufgrund des Profilschnittes könnte für den größten Teil der Struktur - die Basisfläche der kiesigen Bildungen fällt nach Westen hin ein - eine Entwässerung von der großen Randlage (SchiphorstLütjensee) nach Westen, d.h. in das Beckeninnere hinein, angenommen werden. Andererseits zeigen die deutlichen Entwässerungsbahnen am östlichen Rand der Struktur sowie die flächenhaften Schmelzwasserablagerungen in der östlich gelegenen Niederung eine bevorzugte Schüttung von Schmelzwasser nach Osten an. Da in einer Deglaziationsphase verschiedene zeitlich gestaffelte Phasen stattgefunden haben können, ist die Beantwortung dieser Frage späteren detaillierteren Untersuchungen vorbehalten.

Die Anlage einer Esker-Struktur auf einer Eisrandlage ist grundsätzlich ungewöhnlich, da Esker vorwiegend in Tunneltälern oder anderen Talformen vorkommen. Naturgemäß müssen beim Niedertauen von Inlandeismassen aber auch höher gelegene Bereiche entwässert worden sein. Möglicherweise ist die Struktur im Forst Steinburg durch die besonderen Verhältnisse, im Zwickel einer Gletscherzunge und einer benachbarten hoch aufragenden Eisrandlage, besonders gestaltet. Nicht zuletzt die Vergesellschaftung mehrerer parallel angelegter Esker unterschiedlicher Größe lässt die Struktur als seltene Bildung erscheinen, die so in der wissenschaftlichen Literatur bisher nicht beschrieben worden ist.

Das Vorhandensein derartiger Strukturen liefert Hinweise auf die regionale Entwässerung in der letzten Vereisungsphasen einer Region. Damit werden neue Erkenntnisse hinsichtlich der Vereisungsgeschichte möglich. Eine genauere Kartierung ist folglich wünschenswert.

\section{Danksagung}

Frau Carmen Schubert sowie die Herren Gaterman, Fokuhl und Bielfeld (alle Franzdorf) genehmigten freundlicherweise die Durchführung von Rammkernsondierungen. Es ist den Herren Herbert und Ewald Hack ( $\dagger$; Franzdorf) zu verdanken, dass vor einigen Jahrzehnten ein Teil des Eskers vom Abbau ausgespart wurde. Maike Schirk sei für die Erstellung des Profilschnittes gedankt. Sven Lukas (London) danke ich für die Korrektur des Abstracts, den Reviewern für konstruktive Hinweise.

\section{Literatur}

AARIO, R. (1977): Glacial and glaciofluvial sedimentation in Finnish valley environments. - The conference "The River valley as a focus of interdisciplinary research" 21.-23. June 1977, Finland.

Brodzikowski, K. \&. VAN Loon, A.J. (1991): Glacigenic sediments. - Developments in Sedimentology, 49: 674 S.; Amsterdam u.a.

Duve, C. (1961): Schreiben an den Landkreis Stormarn; Betreff: Gemarkung Sprenge, Antrag der Fa. Bock \& Schulte auf Errichtung einer Kieswaschanlage auf der Steinburg. Brief vom 5. Januar 1961 [unveröff.].

EGGERS, W. (1934): Die Oberflächenformen der jungeiszeitlichen Landschaft im südlichen Schleswig und nördlichen Holstein. - Veröff. d. Schleswig-Holst. Universitätsges., 42: 1-120, Breslau.

GAgEL, C. (1923): Der Oszug von Waldhusen-Pöppendorf bei Lübeck. - Jb. Preuß. Geol. L.-A. (f. d. Jahr 1921), 42: 513-515.

GRIPP, K. (1978): Die Entstehung von Geröll-Osern (Esker). - Eiszeitalter u. Gegenwart, 28: 92-108.

GrubE, A. (2008): Geodiversität in Schleswig-Holstein. - Jahresber. LANU 2007/2008: 227-242.

Grube, A. (2010): Sülfelder Tannen - eine einmalige Niedertau- und Kames-Landschaft der Weichsel-Kaltzeit (Kreise Stormarn und Segeberg). - Natur- und Landeskunde, 117 (7-9): 93-101.

Grube, A. (2011): Geotope in Schleswig-Holstein - Dokumentation der Geotope des Landes-Katasters Schleswig-Holstein. - Landesamt für Landwirtschaft, Umwelt und Ländliche Räume Schleswig-Holstein [in Vorbereitung].

Halske, H. (1924): Verlauf der Endmoränen auf dem Meßtischblatt Trittau. - Diss. Univ. Hamburg, 43 S., Hamburg.

JAMIESON, T. F. (1874): On the last stage of the glacial period in North Britain - Quaterly Journal of the Geological Society of London, 30: 317-338.

Kölling, M. \& SchlÜter, M. (1988): Das Ahrensburg-Stellmoorer Tunneltal (Nordostteil). - Meyniana, 41: 85-95.

LUNDQVIST, J. (1979): Morphogentic classification of glaciofluvial deposits. - Sveriges Geologiska Undersökning, Avhandlingar och Uppsatser, 73 (8): 3-72.

OHNEsorge, W. (1928): Der Lübecker Os und seine prähistorischen Altertümer. - Mitt. Geogr. Ges. u.d. Naturhist. Mus. Lübeck, 2 (32): 5-123.

RANGE, P. (1935): Geologische Karte von Preußen 1:25000 - Erläuterungen zu Blatt Eichede; Nr. 839. - 34 S., Preuß. Geol. Landesanstalt, Berlin.

Schulz, W. (1970): Über Oser und osähnliche Bildungen in der westlichen Prignitz. - Jb. Geol., 3: 411-420.

SEIFERT, G. (1953): Das mikroskopische Korngefüge des Geschiebemergels als Abbild der Eisbewegung, zugleich Geschichte des Eisabbaues in Fehmarn, Ost-Wagrien und dem Dänischen Wohld. - Meyniana 2:124-184.

Strehl, E. (1993): Der Wallberg (Os) von Ohe bei Rendsburg. - Die Heimat, 100 (9) 213-217.

Strehl, E. (2006): Die Oser (Wallberge) im Altkreis Eckernförde. - Jahrbuch der Heimatgemeinschaft Eckernförde e.V.; Schwansen, Amt Hütten und Dänischwohld, 64: 249-252.

STRUCK, R. (1918): Wallberge in Lübecks nächster Umgebung. - Lübeckische Blätter, 60 (16): 181-184.

WünNEMANN, B. (1990): Die weichselzeitliche Entstehung der Langseerinne (Angeln) in Schleswig-Holstein. - Diss., Fachbereich Geowissenschaften, FU Berlin, 171 S. + Anhang, Berlin. 